\title{
Law of the Iterated Logarithm for Perturbed Empirical Distribution Functions Evaluated at a Random Point for Nonstationary Random Variables ${ }^{1}$
}

\author{
Michel Hare $^{2}$ and Madan L. Puri ${ }^{3}$
}

Received April 1, 1993; revised October 7, 1993

\begin{abstract}
We consider perturbed empirical distribution functions $\hat{F}_{n}(x)=1 / n \sum_{i=1}^{n} G_{n}\left(x-X_{i}\right)$, where $\left\{G_{n}, n \geqslant 1\right\}$ is a sequence of continuous distribution functions converging weakly to the distribution function of unit mass at 0 , and $\left\{X_{i}, i \geqslant 1\right\}$ is a nonstationary sequence of absolutely regular random variables. We derive the almost sure representation and the law of the iterated logarithm for the statistic $\hat{F}_{n}\left(U_{n}\right)$ where $U_{n}$ is a $U$-statistic based on $X_{1}, \ldots, X_{n}$. The results obtained extend or generalize the results of Nadaraya, ${ }^{(7)}$ Winter, ${ }^{(16)}$ Puri and Ralescu, ${ }^{(9.10)}$ Oodaira and Yoshihara, ${ }^{(8)}$ and Yoshihara, ${ }^{(19)}$ among others.
\end{abstract}

KEY WORDS: Perturbed empirical distribution functions; absolutely regular processes; strong mixing; almost sure representation; $U$-statistic; law of the iterated logarithm.

\section{INTRODUCTION}

Let $\left\{X_{i}, i \geqslant 1\right\}$ be a sequence of absolutely regular r.v.s (random variables) with continuous c.d.f.s (cumulative distribution functions) $\left\{F_{i}, i \geqslant 1\right\}$ defined on the real line $\mathbb{R}$ and let $\widetilde{F}_{n}$ be the corresponding empirical distribution function based on $X_{1}, \ldots, X_{n}$, i.e., $\widetilde{F}_{n}(x)=n^{-1} \sum_{i=1}^{n} u\left(x-X_{i}\right)$ where $u(t)=1$ or 0 according as $t \geqslant 0$ or $t<0$. Suppose that $F_{i}$ converges to a fixed d.f. $F$ as $i \rightarrow \infty$. If $\left\{X_{i}, i \geqslant 1\right\}$ is a stationary sequence (in which case $F_{i} \equiv F$ for all $i$ ), $\tilde{F}_{n}$ is a natural estimator of $F$ based on the sample $X_{1}, \ldots, X_{n}$. However if $F$ is a smooth distribution function, then it seems

\footnotetext{
' Research supported by the Office of Naval Research Contract N00014-91-J-1020.

${ }^{2}$ U.R.A. C.N.R.S. 743, Orsay, France and Indiana University, Bloomington, Indiana, 47405.

${ }^{3}$ Indiana University, Bloomington. Indiana, 47405.
} 
reasonable to consider smooth estimators which are better adapted to this situation. For the case $\left\{X_{i}, i \geqslant 1\right\}$ is a sequence of iid (independent and identically distributed) r.v.s, Watson and Leadbetter, ${ }^{(14)}$ Nadaraya, ${ }^{(7)}$ Yamato, ${ }^{(18)}$ and Winter, ${ }^{(16.17)}$ have considered an estimator of the form

$$
\hat{F}_{n}(x)=n^{-1} \sum_{i=1}^{n} G_{n}\left(x-X_{i}\right), \quad x \in \mathbb{R}, \quad n \geqslant 1
$$

where $G_{n}(x)=\int_{-\infty}^{x} g_{n}(t) d t, g_{n}(t)=\alpha_{n}^{-1} g\left(t \alpha_{n}^{-1}\right),\left\{\alpha_{n} ; n \geqslant 1\right\}$ is a sequence of positive real numbers such that $\alpha_{n} \rightarrow 0$ as $n \rightarrow \infty$, and $g$ is a probability density function. Watson and Leadbetter ${ }^{(14)}$ established the asymptotic normality of $\hat{F}_{n}$, while Nadaraya, ${ }^{(7)}$ Winter, ${ }^{(16)}$ and Yamato, ${ }^{(18)}$ proved the a.s. (almost sure) convergence of $\hat{F}_{n}$ to $F$. Later Winter ${ }^{(17)}$ showed that $\hat{F}_{n}$ has the Chung-Smirnov property, i.e. with probability one,

$$
\limsup _{n \rightarrow \infty}(2 n / \log n)^{1 / 2} \sup _{x}\left|\hat{F}_{n}(x)-F(x)\right| \leqslant 1
$$

For some related results dealing with the estimation of probability density function, see Schuster, ${ }^{(11)}$ Scott et al., ${ }^{(12)}$ and Wertz, ${ }^{(15)}$ for a general review in this area.

Let $h\left(x_{1}, \ldots, x_{m}\right)$, symmetric in its arguments be a measurable kernel (of degree $m$ ), and let $U_{n}$ be the corresponding $U$-statistics given by

$$
U_{n}=\left(\begin{array}{c}
n \\
m
\end{array}\right)^{-1} \sum_{C_{n, m}} h\left(X_{i_{1}}, \ldots, X_{i_{m}}\right)
$$

where $C_{n, m}$ denotes the set of all $\left(\begin{array}{c}n \\ m\end{array}\right)$ combination of $m$ distinct elements $\left\{i_{1}, \ldots, i_{m}\right\}$ from $\{1, \ldots, n\}$. Let

$$
\theta(F)=\int_{\mathbb{R}^{m}} h\left(x_{1}, \ldots, x_{m}\right) \prod_{i=1}^{m} d F\left(x_{i}\right)
$$

When the r.v.s are i.i.d., Puri and Ralescu ${ }^{(10)}$ derived the asymptotic normality of $\hat{F}_{n}\left(U_{n}\right)$ which is useful in estimating $\xi=F(\theta(F))$ when $F$ is unknown. Later Sun ${ }^{(13)}$ extended this result when the r.v.s are stationary and absolutely regular. Lea and Puri ${ }^{(6)}$ established for the iid case, (i) the almost sure (a.s.) representation, (ii) the law of the iterated logarithm and (iii) an invariance principle for $\hat{F}_{n}\left(U_{n}\right)$. In this paper, we extend the results (i) and (ii) when the r.v.s are nonstationary and absolutely regular.

With our general framework (nonstationarity and absolute regularity), the results have applications for a larger class of processes such as ARMA processes and Markov processes for which the initial measure is not 
necessary to be the invariant measure. For example, suppose that the sequence $\left\{X_{i}, i \geqslant 1\right\}$ of r.v.'s is an ARMA process defined by

$$
X_{i}=a X_{i-1}+b \varepsilon_{i}+\varepsilon_{i-1}, \quad i \geqslant 1
$$

where $X_{0}$ admits a strictly positive density, $\left\{\varepsilon_{i}, i \geqslant 1\right\}$ is a sequence of i.i.d. r.v.'s with strictly positive density such that $E\left(\varepsilon_{i}\right)=0$ and $a$ and $b$ are real numbers such that $|a|<1$. Then, from Harel and Puri, ${ }^{(4)}$ this process is absolutely regular and for any initial measure, the conclusions of the theorems and propositions here hold if the sequence of r.v.'s is an ARMA process defined by Eq. (1.4). The results of our paper can also be applied for nonstationary Markov processes (see, e.g. Harel and Puri ${ }^{(5)}$ ).

\section{SOME PRELIMINARY RESULTS}

In the following, we suppose that the sequence $\left\{X_{i}, i \geqslant 1\right\}$ is absolutely regular with rates

$$
\beta(m)=O\left(\rho^{m}\right), \quad 0<\rho<1
$$

Recall that a nonstationary sequence $\left\{X_{n i}, 1 \leqslant i \leqslant n, n \geqslant 1\right\}$ is absolutely regular if

$$
\begin{aligned}
& \sup _{m \leqslant n} \max _{1 \leqslant j \leqslant n-m} E\left\{\sup _{A \in \sigma\left(X_{n i}, i \geqslant j+m\right)}\left|P\left(A \mid \sigma\left(X_{n i}, 1 \leqslant i \leqslant j\right)\right)-P(A)\right|\right\} \\
& =\beta(m) \downarrow 0 \text { as } m \rightarrow \infty
\end{aligned}
$$

Here $\sigma\left(X_{n i}, 1 \leqslant i \leqslant j\right)$ and $\sigma\left(X_{n i}, i \geqslant j+m\right)$ are the $\sigma$-fields generated by $\left(X_{n 1}, \ldots, X_{n j}\right)$ and $\left(X_{n, j+m}, X_{n, j+m+1}, \ldots, X_{m m}\right)$ respectively. Also recall that $\left\{X_{n i}\right\}$ satisfies the strong mixing condition if $\sup _{m \leqslant n} \sup _{1 \leqslant j \leqslant n-m}\{\mid P(A \cap B)$ $\left.-P(A) P(B) \mid ; A \in \sigma\left(X_{n i}, 1 \leqslant i \leqslant j\right), \quad B \in \sigma\left(X_{n i}, i \geqslant j+m\right)\right\}=\alpha(m) \downarrow 0$ as $m \rightarrow \infty$. Since $\alpha(m) \leqslant \beta(m)$, it follows that if $\left\{X_{n i}\right\}$ is absolutely regular, then it is also strong mixing.

Let $\left\{d_{n}\right\}$ be a sequence of positive real numbers such that $d_{n} \sim k n^{-1 / 2}(\log \log n)^{1 / 2}$ as $n \rightarrow \infty$, for some $k>0$. Define

$$
K_{n}(t)=\sup _{|x| \leqslant d_{n}}\left|\tilde{F}_{n}(t+x)-\tilde{F}_{n}(t)-\bar{F}_{n}(t+x)-\bar{F}_{n}(t)\right|
$$

where $\bar{F}_{n}=n^{-1} \sum_{i=1}^{n} F_{i}$, and let $F_{i}^{\prime}$ denote the density of $F_{i}$.

Lemma 2.1. For $\delta>0$, if $\left\{F_{i}^{\prime}, i \geqslant 1\right\}$ is uniformly bounded in $(\theta(F)-\delta, \theta(F)+\delta)$ and

$$
K_{n}^{*}=\sup _{|t-\theta(F)|<\delta / 2} K_{n}(t)
$$


then, for any $v\left(0<v<\frac{1}{8}\right)$ and for any $s>1$ there exist two positive constants $C_{v}^{(1)}$ and $C_{v}^{(2)}$ and an integer $n_{s}$ such that

$$
P\left[K_{n}^{*} \geqslant C_{v}^{(1)} n^{-3 / 4+v}\right] \leqslant C_{v}^{(2)} n^{-s}
$$

for all $n \geqslant n_{s}$. Hence

$$
K_{n}^{*}=O\left(n^{-3 / 4+v}\right) \text { a.s. as } n \rightarrow \infty
$$

Proof. We start in a way similar way to Lemma 2.1 of Lea and Puri. ${ }^{(6)}$ For $\eta \in[\theta(F)-\delta / 2, \theta(F)+\delta / 2]$, let

$$
H_{n}(x)=\tilde{F}_{n}(x)-\tilde{F}_{n}(\eta)-\bar{F}_{n}(x)+\bar{F}_{n}(\eta)
$$

Let $\left\{b_{n}, n \geqslant 1\right\}$ be a sequence of positive integers such that $b_{n} \sim n^{1 / 4}$ as $n \rightarrow \infty$. Consider a particular $n$. For any integer $r \in\left[-b_{n}, b_{n}\right]$, let $\eta_{r, n}=\eta+d_{n} b_{n}^{-1} r$ and $\theta_{r, n}=\bar{F}_{n}\left(\eta_{r+1, n}\right)-\bar{F}_{n}\left(\eta_{r, n}\right)$. Since $\tilde{F}_{n}$ and $\bar{F}_{n}$ are nondecreasing, it follows from Eq. (2.4) that for $x \in\left[\eta_{r, n}, \eta_{r+1, n}\right]$,

$$
H_{n}(x) \leqslant \widetilde{F}_{n}\left(\eta_{r+1, n}\right)-\widetilde{F}_{n}(\eta)-\bar{F}_{n}\left(\eta_{r, n}\right)+\bar{F}_{n}(\eta)=H_{n}\left(\eta_{r+1, n}\right)+\theta_{r, n}
$$

Similarly

$$
H_{n}(x) \geqslant H_{n}\left(\eta_{r, n}\right)-\theta_{r, n} \text { for } x \in\left[\eta_{r, n}, \eta_{r+1, n}\right]
$$

It follows from Eqs. (2.1), (2.5), and (2.6) that

$$
K_{n}(\eta) \leqslant J_{n}+\beta_{n}
$$

where

$$
J_{n}=\max _{-b_{n} \leqslant r \leqslant b_{n}}\left|H_{n}\left(\eta_{r, n}\right)\right|, \quad \beta_{n}=\max _{-b_{n} \leqslant r \leqslant b_{n}} \theta_{r, n}
$$

Now, since $\left\{F_{i}, i \geqslant 1\right\}$ is uniformly bounded in a neighborhood of $\eta$, $\bar{F}_{n}^{\prime}$ is also uniformly bounded in a neighborhood of $\eta$, it follows that for large $n$

$$
\beta_{n}=O\left(n^{-3 / 4}(\log \log n)^{1 / 2}\right)
$$

Let $C_{2}$ be the upper bound of $\sup _{n \geqslant 1} \bar{F}_{n}^{\prime}(x)$ in $(\xi-\delta, \xi+\delta)$. Since $d_{n} \rightarrow 0$ as $n \rightarrow \infty$, there exists a positive integer $N_{1}$ such that $\sup _{n \geqslant 1} \bar{F}_{n}(\eta)-\bar{F}_{n}\left(\eta-d_{n}\right) \leqslant C_{2} d_{n}$ for all $n \geqslant N_{1}$. Applying Lemma 5.2 (see Appendix), with $m=n^{2 v-\varepsilon}, 0<\varepsilon<2 v$, and $a=\frac{7}{8}$, we have

$$
P\left[\left|H_{n}\left(\eta_{r, n}\right)\right| \geqslant \delta_{n}^{(v)}\right] \leqslant 2 n^{2 v-\varepsilon} \exp \left\{-r_{n}^{(v)}\right\}+2 n \beta\left(\left[n^{2 v-\varepsilon}\right]\right)
$$


where $\delta_{n}^{(v)}=C_{1} n^{-3 / 4+v}$, [a] is the integer part of $a, C_{1}$ is some constant $>0$ and

$$
r_{n}^{(v)}=n^{7 / 8^{(1-2 c+c)}} \delta_{n}^{(v)} / 5 M_{0}
$$

After some routine computations, we obtain

$$
r_{n}^{(v)}=O\left(n^{1 / 4-v+\varepsilon}\right)
$$

which implies

$$
n^{2 v-\varepsilon} \exp \left\{-r_{n}^{(v)}\right\}=O\left(n^{-(s+3 / 4)}\right)
$$

We also have

$$
\beta\left(\left[n^{2 v-\varepsilon}\right]\right)=O\left(e^{(\log \rho) n^{2 v-\varepsilon}}\right)
$$

Thus, from Eq. (2.10), we deduce

$$
P\left[\left|H\left(\eta_{r, n}\right)\right| \geqslant \delta_{n}^{(v)}\right]=O\left(n^{-(s+3 / 4)}\right)+O\left(n e^{n^{(2 v-\varepsilon)} \log \rho}\right)
$$

It follows from Eqs. (2.8) and (2.14) that

$$
P\left[J_{n} \geqslant \delta_{n}^{(v)}\right]=O\left(b_{n} n^{-(s+3 / 4)}\right)+O\left(b_{n} n e^{p^{(2 v-c)} \log \rho}\right)
$$

It is easy to see that if $C_{1}$ is chosen sufficiently large, there exists a positive constant $C_{2}$ and a positive integer $N_{1}$ such that for $n \geqslant N_{1}$

$$
P\left[J_{n} \geqslant \delta_{n}^{(v)}\right] \leqslant C_{2} n^{-(s+1 / 2)}
$$

From Eqs. (2.7), (2.9), and (2.16), it follows that there exists a positive integer $N_{2}$ such that

$$
P\left[K_{n}(\eta) \geqslant 2 \delta_{n}^{(v)}\right] \leqslant C_{2} n^{-(s+1 / 2)} \quad \text { for } n \geqslant N_{2}
$$

Now, let

$$
r_{j, n}=\theta(F)+\frac{j \delta}{2 \sqrt{n}}, \quad j=-[\sqrt{n}], \ldots, 0, \ldots,[\sqrt{n}]
$$

Then by Eq. (2.17), we have

$$
\begin{aligned}
P\left[\max _{-\sqrt{n} \leqslant j \leqslant \sqrt{n}} K_{n}\left(r_{j, n}\right) \geqslant 2 \delta_{n}^{(v)}\right] & \leqslant 2 C_{2} n^{1 / 2} n^{-(s+1 / 2)} \\
& =2 C_{2} n^{-s} \text { for } n \geqslant N_{2}
\end{aligned}
$$


For any $t \in[\theta(F)-\delta / 2, \theta(F)+\delta / 2]$ and for any $x \in\left[-d_{n}, d_{n}\right]$ where $x \neq 0$, there exist two integers $j_{1}$ and $j_{2}$ such that

$$
[-\sqrt{n}] \leqslant j_{1}<j_{2} \leqslant[\sqrt{n}]
$$

and

$$
\begin{aligned}
\theta(F)+\frac{\left(j_{1}-1\right) \delta}{2 \sqrt{n}} & <\underline{t} \leqslant \theta(F)+\frac{j_{1} \delta}{2 \sqrt{n}} \leqslant \theta(F)+\frac{\left(j_{2}-1\right) \delta}{2 \sqrt{n}} \\
& <i \leqslant \theta(F)+\frac{j_{2} \delta}{2 \sqrt{n}}
\end{aligned}
$$

where $\underline{t}=\min \{t, t+x\}$ and $\bar{t}=\max \{t, t+x\}$.

From the triangular inequality, we obtain

$$
\begin{aligned}
\left|V_{n}(t)-V_{n}(t+x)\right| \leqslant & \left|V_{n}(t)-V_{n}\left(\theta(F)+\frac{j_{1} \delta}{2 \sqrt{n}}\right)\right| \\
& +\left|V_{n}\left(\theta(F)+\frac{j_{1} \delta}{2 \sqrt{n}}\right)-V_{n}\left(\theta(F)+\frac{\left(j_{2}-1\right) \delta}{2 \sqrt{n}}\right)\right| \\
& +\left|V_{n}\left(\theta(F)+\frac{\left(j_{2}-1\right) \delta}{2 \sqrt{n}}\right)-V_{n}(t+x)\right|
\end{aligned}
$$

where $V_{n}(t)=\tilde{F}_{n}(t)-\bar{F}_{n}(t)$.

As $d_{n} \geqslant \delta / 2 \sqrt{n}$ for $n$ sufficiently large, we deduce

$$
K_{n}^{*} \leqslant 3 \max _{-\sqrt{n} \leqslant j \leqslant \sqrt{n}} K_{n}\left(r_{j, n}\right)
$$

Choosing $C_{v}^{(1)}=6 C_{1}, C_{v}^{(2)}=2 C_{2}$ and $n \geqslant N_{2}$, it follows, from Eqs. (2.18) and (2.19), that

$$
P\left[K_{n}^{*} \geqslant C_{v}^{(1)} n^{-3 / 4+v}\right] \leqslant C_{v}^{(2)} n^{-s}
$$

Thus Eq. (2.3) follows. Also

$$
\sum_{n=1}^{\infty} P\left[K_{n}^{*} \geqslant C_{v}^{(1)} n^{-3 / 4+v}\right] \leqslant C_{v}^{(2)} \sum_{n=1}^{\infty} n^{-s}<\infty
$$

Consequently, using the Borel-Cantelli Lemma, we obtain

$$
K_{n}^{*}=O\left(n^{-3 / 4+v}\right) \text { a.s. } \quad \text { as } n \rightarrow \infty
$$

This completes the proof. 
Lemma 2.2. If (i) $\left\{F_{i}^{\prime}, i \geqslant 1\right\}$ is uniformly bounded in a neighborhood of $\theta(F)$ and (ii) $\int x^{2} g(x) d x<\infty$, then

$$
\begin{aligned}
\left|\hat{F}_{n}(\theta(F))-\widetilde{F}_{n}(\theta(F))\right|= & O\left(n^{-3 / 4+v}\right) \\
& +O\left(n \alpha_{n}^{2}(\log \log n)^{-1}\right) \text { a.s. }\left(0<v<\frac{1}{8}\right)
\end{aligned}
$$

Proof. We proceed as in Lemma 2.2 of Lea and Puri. ${ }^{(6)}$ First note that

$$
\begin{aligned}
\hat{F}_{n}(\theta(F)) & =n^{-1} \sum_{i=1}^{n} G_{n}\left(\theta(F)-X_{i}\right)=\int_{-\infty}^{+\infty} G_{n}(\theta(F)-t) d \widetilde{F}_{n}(t) \\
& =\int_{-\infty}^{+\infty} \tilde{F}_{n}(\theta(F)-t) g_{n}(t) d t
\end{aligned}
$$

and

$$
\int_{-\infty}^{+\infty} g_{n}(t) d t=1
$$

Condition (i) ensures that there exists $\delta>0$ and $M>0$ such that $\sup _{i \in \mathbb{N}}\left|F_{i}(\theta(F)-t)-F_{i}(\theta(F))\right| \leqslant M|t|$ for all $|t|<\delta$.

Using Lemma 2.1 and Eqs. (2.20) and (2.21), we have

(a) $\left|\hat{F}_{n}(\theta(F))-\tilde{F}_{n}(\theta(F))\right|$

$$
\begin{aligned}
\leqslant & \int_{-\infty}^{+\infty}\left|\widetilde{F}_{n}(\theta(F)-t)-\widetilde{F}_{n}(\theta(F))\right| g_{n}(t) d t \\
\leqslant & \int_{|t| \leqslant d_{n}}\left|\widetilde{F}_{n}(\theta(F)-t)-\widetilde{F}_{n}(\theta(F))\right| g_{n}(t) d t \\
& +\int_{|t|>d_{n}}\left|\widetilde{F}_{n}(\theta(F)-t)-\tilde{F}_{n}(\theta(F))\right| g_{n}(t) d t \\
\leqslant & \int_{|r| \leqslant d_{n}}\left|\bar{F}_{n}(\theta(F)-t)-\bar{F}_{n}(\theta(F))\right| g_{n}(t) d t \\
& +\int_{|t|>d_{n}} g_{n}(t) d t+O\left(n^{-3 / 4+v}\right) \text { a.s. }
\end{aligned}
$$

For large $n$, the first term on the right-hand side of the last inequality in (a) is bounded by

(b) $\quad M \int_{-\infty}^{+\infty}|t| g_{n}(t) d t=M \alpha_{n} \int_{-\infty}^{+\infty}|y| g(y) d y=O\left(\alpha_{n}\right)$ 
and the second term is bounded by

(c) $n \alpha_{n}^{2}(\log \log n)^{-1} \int_{|y|>d_{n} / x_{n}} y^{2} g(y) d y=O\left(n \alpha_{n}^{2}(\log \log n)^{-1}\right)$

since $\int_{-\infty}^{+\infty} y^{2} g(y) d y<\infty$. Thus, from (a)-(c) we have

$$
\begin{aligned}
\left|\hat{F}_{n}(\theta(F))-\widetilde{F}_{n}(\theta(F))\right| & =O\left(\alpha_{n}\right)+O\left(n \alpha_{n}^{2}(\log \log n)^{-1}\right)+O\left(n^{-3 / 4+v}\right) \\
& =O\left(n \alpha_{n}^{2}(\log \log n)^{-1}\right)+O\left(n^{-3 / 4+v}\right)
\end{aligned}
$$

a.s. as $n \rightarrow \infty$ and Lemma 2.2 is proved.

Because of the hypothesis of nonstationarity, we have to define some new notations for the $U$-statistics.

For every $p(1 \leqslant p \leqslant m)$ and $n \geqslant m$, let $1 \leqslant i_{1} \neq \cdots \neq i_{p} \leqslant n$ be arbitrary integers. Put

$$
h_{p, n}^{\left(i_{1}, \ldots, i_{n}\right)}\left(x_{1}, \ldots, x_{p}\right)=\sum_{\left(i_{p+1}, \ldots, i_{m}\right\} \in I_{p, n}\left(i_{1}, \ldots, i_{p}\right)} \lambda\left(x_{1}, \ldots, x_{p} ; i_{p+1}, \ldots, i_{m}\right)
$$

where

$$
\lambda\left(x_{1}, \ldots, x_{p} ; i_{p+1}, \ldots, i_{m}\right)=\int_{\mathbb{R}^{m-p}} h\left(x_{1}, \ldots, x_{m}\right) d F_{i_{p+1}}\left(x_{p+1}\right) \ldots d F_{i_{m}}\left(x_{m}\right)
$$

and $\quad I_{p, n}\left(i_{1}, \ldots, i_{p}\right)=\left\{\left(i_{1}, \ldots, i_{p}\right) ; \quad 1 \leqslant i_{p+1} \neq \ldots \neq i_{m} \leqslant n, \quad i_{l} \notin\left(i_{1}, \ldots, i_{p}\right)\right.$, $p+1 \leqslant l \leqslant m\}$ and

$$
h_{0, n}=\sum_{\left(i_{1}, \ldots, i_{m 1}\right\} \in I_{0, n}} \int_{\mathbb{R}^{m}} h\left(x_{1}, \ldots, x_{m}\right) d F_{i_{1}}\left(x_{1}\right), \ldots, d F_{i_{m}}\left(x_{m}\right)
$$

where $I_{0, n}=\left\{\left(i_{1}, \ldots, i_{m}\right), 1 \leqslant i_{1} \neq \ldots \neq i_{m} \leqslant n\right\}$.

For every $p(1 \leqslant p \leqslant m)$, set

$$
\begin{aligned}
U_{n}^{(p)}= & n^{-[m]} \sum_{1 \leqslant i_{1} \neq \cdots \neq i_{p} \leqslant n} \int_{\mathbb{R}^{p}} h_{p, n}^{\left(i_{1}, \ldots, i_{p}\right)}\left(x_{1}, \ldots, x_{p}\right) \\
& \times \prod_{j=1}^{p} d\left(I_{\left[x_{i j} \leqslant x_{j}\right]}-F_{i j}\left(x_{j}\right)\right)
\end{aligned}
$$

where $n^{-[m]}=(n(n-1) \cdots(n-m+1))^{-1}$. 
Lemma 2.3. If there exists a positive number $\delta$ such that for $r=4+\delta$

$$
\begin{aligned}
& \sup _{n} \max _{\left(i_{1}, \ldots, i_{m}\right) \in\{1, \ldots, n\}^{m}} \int_{\mathbb{R}^{m}}\left|h\left(x_{1}, \ldots, x_{m}\right)\right|^{r} d F_{i_{1}}\left(x_{1}\right) \\
& \times \cdots \times d F_{i_{m}}\left(x_{m}\right) \leqslant M_{0}<\infty \\
& \sup _{n} \max _{\left(i_{1}, \ldots, i_{m}\right) \in\{1, \ldots, n\}^{m}} E\left(\left|h\left(X_{i_{1}}, \ldots, X_{i_{m}}\right)\right|^{r}\right) \leqslant M_{0}<\infty
\end{aligned}
$$

and for some $\delta^{\prime}\left(0<\delta^{\prime}<\delta\right), \beta(n)=O\left(n^{-3\left(4+\delta^{\prime}\right) /\left(2+\delta^{\prime}\right)}\right)$, then we have

$$
E\left(U_{n}^{(2)}\right)^{4}=O\left(n^{-3-\gamma}\right)
$$

where $\gamma=6\left(\delta-\delta^{\prime}\right) /(4+\delta)\left(2+\delta^{\prime}\right)>0$ and

$$
E\left(U_{n}^{(p)}\right)^{2}=O\left(n^{-3}\right), \quad 3 \leqslant p \leqslant m
$$

Proof. We first note that

$$
\begin{aligned}
U_{n}^{(2)=} & n^{-[m]} \sum_{1 \leqslant i_{1} \neq i_{2} \leqslant n} \int_{\mathbb{R}^{2}} h_{2, n}^{\left(i_{1}, i_{2}\right)}\left(x_{1}, x_{2}\right) d\left(I_{\left[x_{i_{1}} \leqslant x_{1}\right]}\right. \\
& \left.-F_{i_{1}}\left(x_{1}\right)\right) d\left(I_{\left[x_{i_{2}} \leqslant x_{2}\right]}-F_{i_{2}}\left(x_{2}\right)\right)
\end{aligned}
$$

So we have

$$
\begin{aligned}
E\left(U_{n}^{(2)}\right)^{4}= & \left(n^{-[m]}\right)^{4} \sum_{1 \leqslant i_{11}, i_{12} \leqslant n} \sum_{1 \leqslant i_{21}, i_{22} \leqslant n} \sum_{1 \leqslant i_{11}, i_{32} \leqslant n} \\
& \times \sum_{1 \leqslant i_{41}, i_{42} \leqslant n} J\left(\left(i_{11}, i_{12}\right),\left(i_{21}, i_{22}\right),\left(i_{31}, i_{32}\right),\left(i_{41}, i_{42}\right)\right)
\end{aligned}
$$

where

$$
\begin{aligned}
& J\left(\left(i_{11}, i_{12}\right),\left(i_{21}, i_{22}\right),\left(i_{31}, i_{32}\right),\left(i_{41}, i_{42}\right)\right) \\
& =E\left\{\prod_{j=1}^{4} \int_{\mathbb{R}^{2}} h_{2, n}^{\left(i_{1}, i_{2}\right)}\left(x_{j 1}, x_{j 2}\right) d\left(I_{\left[x_{j_{1}} \leqslant x_{j 1}\right]}-F_{i_{11}}\left(x_{j 1}\right)\right) d\left(I_{\left[x_{i j 2} \leqslant x_{j 2}\right]}-F_{i_{j 2}}\left(x_{j 2}\right)\right)\right\}
\end{aligned}
$$

and

$$
h_{2, n}^{\left(i_{1} 1, i_{j}\right)}\left(x_{i 1}, x_{i 2}\right)=\sum_{\left(i_{3}, \ldots, i_{m}\right) \in I_{2, n}\left(i_{1}, i_{j}\right)} g\left(x_{i 1}, x_{i 2}, x_{3}, \ldots, x_{m}\right) d F_{i_{3}}\left(x_{3}\right) \cdots d F_{i_{m}}\left(x_{m}\right)
$$


Let $i_{r s}(\leqslant n)(r=1, \ldots, 4, s=1,2)$ be mutually different. Reorder $i_{r s}$ as $1 \leqslant k_{1}<k_{2}<\cdots<k_{8} \leqslant n$ and put

$$
J\left(\left(i_{11}, i_{12}\right), \ldots,\left(i_{41}, i_{42}\right)\right)=H\left(k_{1}, \ldots, k_{8}\right)=E\left[g\left(X_{k_{1}}, \ldots, X_{k_{8}}\right)\right]
$$

Let $d^{(c)}$ be the $c$ th largest difference among $\left(k_{j+1}-k_{j}\right), j=1, \ldots, 7$

Since

$$
\int_{\mathbb{R}^{8}} g\left(x_{1}, \ldots, x_{8}\right) P_{j}^{\left(k_{1} \ldots, \ldots k_{8}\right)}\left(x_{1}, \ldots, x_{8}\right)=0(j=1,7) \text { (see Appendix) }
$$

It follows from Lemma 5.1 (see Appendix) that

$$
\begin{aligned}
H\left(k_{1}, \ldots, k_{8}\right) \leqslant & \left(\frac{n^{[m]}}{n(n-1)}\right)^{4} M_{0}\left[\beta\left(k_{8}-k_{7}\right)\right]^{(2+\delta) /(4+\delta)} \\
& \text { if } k_{8}-k_{7}=d^{(1)}
\end{aligned}
$$

and

$$
\begin{aligned}
H\left(k_{1}, \ldots, k_{8}\right) \leqslant & \left(\frac{n^{[m]}}{n(n-1)}\right)^{4} M_{0}\left[\beta\left(k_{2}-k_{1}\right)\right]^{(2+\delta) /(4+\delta)} \\
& \text { if } k_{2}-k_{1}=d^{(1)}
\end{aligned}
$$

Hence

$$
\begin{aligned}
& \sum_{\substack{1 \leqslant k_{1}<\ldots<k_{8} \leqslant n \\
k_{8}-k_{7}=d^{(1)} \text { or } k_{2}-k_{1}=d^{(1)}}} H\left(k_{1}, \ldots, k_{8}\right) \\
& \leqslant\left(\frac{n^{[m]}}{n(n-1)}\right)^{4} M_{0} n^{4} \sum_{j=1}^{n}(j+1)^{3}[\beta(j)]^{(2+\delta) /(4+\delta)}
\end{aligned}
$$

If for some $j_{\alpha}\left(2 \leqslant j_{\alpha} \leqslant 6,1 \leqslant \alpha \leqslant 4\right), k_{j_{x}+1}-k_{j_{\alpha}}=d^{(\alpha)}(1 \leqslant \alpha \leqslant 4)$, then from Lemma 5.1 (see Appendix)

$$
H\left(k_{1}, \ldots, k_{8}\right) \leqslant\left(\frac{n^{[m]}}{n(n-1)}\right)^{4} M \sum_{\alpha=1}^{4}\left[\beta\left(k_{j_{x}+1}-k_{j_{2}}\right)\right]^{(2+\delta) /(4+\delta)}
$$

and hence

$$
\begin{aligned}
& \sum_{\substack{1 \leqslant k_{1}<\cdots<k_{8} \leqslant n \\
k_{j_{\alpha}+1}-k_{j_{2}}=d^{(x)}(1 \leqslant \alpha \leqslant 4)}} H\left(k_{1}, \ldots, k_{8}\right) \\
& \leqslant 4\left(\frac{n^{[m]}}{n(n-1)}\right)^{4} M_{0} n^{4} \sum_{j=1}^{n}(j+1)^{3}[\beta(j)]^{(2+\delta) /(4+\delta)}
\end{aligned}
$$

Consequently 


$$
\begin{aligned}
& \sum_{1 \leqslant k_{1}<\cdots<k_{8} \leqslant n} H\left(k_{1}, \ldots, k_{8}\right) \\
& \leqslant M\left(\frac{n^{[m]}}{n(n-1)}\right)^{4} n^{4} \sum_{j=1}^{n}(j+1)[\beta(j)]^{(2+\delta) /(4+\delta)}=O\left(n^{4 m-3-\gamma}\right)
\end{aligned}
$$

where $M>0$ is some constant.

We can use a similar method to estimate the sums in the other cases and so we have Eq. (2.26).

The proof of Eq. (2.27) is analogous and so it is omitted.

Lemma 2.4. Under the conditions of Lemma 2.3, we have

$$
U_{n}-\theta(F)=m U_{n}^{(1)}+R_{n}
$$

where $R_{n}=O\left(\left(n^{-1} \log \log n\right)^{1 / 2}\right)$ a.s. as $n \rightarrow \infty$.

Proof. We rewrite Eq. (1.2) as

$$
U_{n}=\theta(F)+\sum_{p=1}^{m}\left(\begin{array}{c}
m \\
p
\end{array}\right) U_{n}^{(p)}=\theta(F)+m U_{n}^{(1)}+\sum_{p=2}^{m}\left(\begin{array}{c}
m \\
p
\end{array}\right) U_{n}^{(p)}
$$

then we have to prove that for every $\varepsilon>0$

$$
P\left[\left|W_{n}\right|>\varepsilon(n \log \log n)^{1 / 2} \text { i.o. }\right]=0
$$

where $\quad W_{n}=n\left(U_{n}-\theta(F)-m U_{n}^{(1)}\right)$. Let $n_{k}=\left[k^{\left(2+\delta^{\prime}\right)(4+\delta) / 3\left(\delta-\delta^{\prime}\right)}\right] \quad$ and $n_{k_{0}} \geqslant m$. Then, from Lemma 2.3 and the Bonferroni inequality

$$
\begin{aligned}
& \sum_{k=k_{0}}^{\infty} P\left[\max _{m \leqslant n \leqslant n_{k}}\left|W_{n}\right| \geqslant \varepsilon\left(n_{k} \log \log n_{k}\right)^{1 / 2}\right] \\
& \leqslant \sum_{k=k_{0}}^{\infty}\left(\sum_{n=m}^{n_{k}} P\left[\left|W_{n}\right| \geqslant \varepsilon\left(n_{k} \log \log n_{k}\right)^{1 / 2}\right]\right) \\
& \leqslant M \sum_{k=k_{0}}^{\infty} k^{-2}<\infty
\end{aligned}
$$

where $M>0$ is some constant. Consequently, from the Borel-Cantelli Lemma, we have

$$
\begin{aligned}
P\left[\left|W_{n}\right|\right. & \left.\geqslant \varepsilon(n \log \log n)^{1 / 2} \text { i.o. }\right] \\
& \leqslant P\left[\max _{n_{k} \leqslant n \leqslant n_{k+1}}\left|W_{n}\right| \geqslant \varepsilon\left(n_{k} \log \log n_{k}\right) \text { i.o. }\right] \\
& \leqslant P\left[\max _{m \leqslant n \leqslant n_{k+1}}\left|W_{n}\right|>\frac{\varepsilon}{4}\left(n_{k+1} \log \log n_{k+1}\right) \text { i.o. }\right]=0
\end{aligned}
$$




\section{A LAW OF THE ITERATED LOGARITHM FOR THE} NONSTATIONARY $U$-STATISTIC

$$
\begin{aligned}
\sigma^{2}\left(\left\{F_{l}^{*}\right\}\right)= & \sigma^{2}=\left[\int_{\mathbb{R}^{m}} h^{2}\left(x_{1}, \ldots, x_{m}\right) \prod_{l=1}^{k} d F\left(x_{l}\right)-\theta^{2}(F)\right] \\
& +2 \sum_{i=1}^{\infty}\left[\int_{\mathbb{R}^{2 m}} h\left(x_{1}, \ldots, x_{m}\right) h\left(x_{m+1}, \ldots, x_{2 m}\right) d F_{i}^{*}\left(x_{1}, x_{m+1}\right)\right. \\
& \left.\times \prod_{l=2}^{m} d F\left(x_{l}\right) \prod_{p=m+2}^{2 m} d F\left(x_{p}\right)-\theta^{2}(F)\right]
\end{aligned}
$$

if the limit exists and is finite.

Let $F_{i, j}$ be the distribution function of $\left(X_{i}, X_{j}\right), 1 \leqslant i<j$.

Theorem 3.1. Suppose the sequence $\left\{X_{i}, i \geqslant 1\right\}$ is absolutely regular with rates satisfying Eq. (2.1). Furthermore, assume that for any $l>1$, there exists a continuous d.f. $F_{I}^{*}$ on $\mathbb{R}^{2}$ with marginals $F$ such that

$$
\left\|F_{i, j}-F_{j-i}^{*}\right\|=O\left(\rho_{0}^{\max (i, j-i)}\right), \quad 1 \leqslant i<j
$$

for some $0<\rho_{0}<1$ where \|\| denotes the norm of total variation.

Suppose also, that there exists a $\delta^{\prime}$ such that Eqs. (2.24) and (2.25) are satisfied. Then we have

$$
\limsup _{n \rightarrow \infty} \frac{n^{1 / 2}\left|U_{n}-\theta(F)\right|}{2^{1 / 2} m \sigma(\log \log n)^{1 / 2}}=1 \text { a.s. }
$$

Proof. From Lemma 2.4, we have only to prove that

$$
\limsup _{n \rightarrow \infty} \frac{\mid U_{n}^{(1)}}{2^{1 / 2} \sigma(\log \log n)^{1 / 2}}=1 \text { a.s. }
$$

where $U_{n}^{(1)}=n^{-1} \sum_{i=1}^{n} X_{n i}^{*}$ and

$$
X_{n i}^{*}=n^{-[m-1]}\left(h_{1, n}^{(i)}\left(X_{i}\right)-\int_{\mathbb{R}} h_{1, n}^{(i)}(x) d F_{i}(x)\right)
$$

From Lemma 5.6 (in the Appendix), we have to verify that the sequence $X_{n i}^{*}$ satisfies the conditions in Eqs. (5.12)-(5.14). Condition in Eq. (5.13) follows from condition in Eq. (2.24). Condition in Eq. (5.14) follows from condition in Eq. (2.1). 
We have to prove condition in Eq. (5.12), that is, for any $n, m$ such that $n \geqslant m$ and any $J \subset\{1, \ldots, n\}$ such that card $J=m$

$$
E\left(\sum_{i \in J} X_{n i}^{*}\right)^{2}=m \sigma^{2}(1+o(1))
$$

We prove Eq. (3.6) for card $J=n$, because for card $J<n$, the proof is similar. Denote

$$
\begin{aligned}
\rho(1)= & \int_{\mathbb{R}^{m}} h^{2}\left(x_{1}, \ldots, x_{m}\right) \prod_{l=1}^{m} d F\left(x_{i}\right)-\theta^{2}(F) \\
\rho(i)=2 & {\left[\int_{\mathbb{R}^{m}} h\left(x_{1}, \ldots, x_{m}\right) h\left(x_{m+1}, \ldots, x_{2 m}\right) d F_{i}^{*}\left(x_{1}, x_{m+1}\right)\right.} \\
& \left.\times \prod_{l=2}^{m} d F\left(x_{l}\right) \prod_{p=m+2}^{2 m} d F\left(x_{p}\right)-\theta^{2}(F)\right], \quad \forall i \geqslant 2 \\
\psi(i, i)= & \int_{\mathbb{R}}\left(h_{1, n}^{(i)}(x)\right)^{2} d F_{i}(x)-\left(\int_{\mathbb{R}} h_{1, n}^{(i)}(x) d F_{i}(x)\right)^{2}, \quad \forall i \geqslant 1 \\
\psi(i, j)= & 2 \int_{\mathbb{R}^{2}}\left(h_{1, n}^{(j)}(x) h_{1, n}^{(j+i)}(y)\right) d F_{i, i+j}(x, y) \\
& -\left(\int_{\mathbb{R}} h_{1, n}^{(j)}(x) d F_{j}(x)\right)\left(\int_{\mathbb{R}} h_{1, n}^{(i+j)}(y) d F_{i+j}(y)\right), \quad i<j
\end{aligned}
$$

Then

$$
\begin{aligned}
\mid E( & \left.\left(\sum_{i=1}^{n} X_{n i}^{*}\right)^{2} / n\right)-\sigma^{2} \mid \\
= & \left|\frac{1}{n}\left(n^{-[m-1]}\right)^{2} \sum_{i=0}^{n-1} \sum_{j=1}^{n-i} \psi(i, j)-\sum_{i=1}^{\infty} \rho(i)\right| \\
\leqslant & \left|\frac{1}{n}\left(n^{-[m-1]}\right)^{2} \sum_{i=0}^{n-1} \sum_{j=1}^{n-i} \psi(i, j)-\frac{1}{n} \sum_{i=1}^{\infty}(n-i) \rho(i)\right| \\
& +\sum_{i=n+1}^{\infty}|\rho(i)|+\sum_{i=1}^{n} \sum_{k=i}^{\infty}|\rho(k)| \\
= & \left|A_{n}\right|+B_{n}+C_{n} .
\end{aligned}
$$

From condition in Eq. (3.2), it follows that $\left|A_{n}\right|=o(1)$.

From condition in Eq. (2.24), we deduce that $|\rho(i)| \leqslant$ 
$(\alpha(i))^{\delta^{\prime} /\left(2+\delta^{\prime}\right)} M_{0}^{2 /\left(2+\delta^{\prime}\right)}$ which implies, by using condition in Eq. (2.1), that $B_{n} \rightarrow 0$ and $C_{n} \rightarrow 0$ as $n \rightarrow \infty$. Hence we have $\left|E\left(\sum_{i=1}^{n} X_{n i}^{*}\right)^{2} / n-\sigma^{2}\right|=o(1)$ and Theorem 3.1 is proved.

\section{A LAW OF THE ITERATED LOGARITHM AND ALMOST SURE REPRESENTATION FOR $\boldsymbol{F}_{n}\left(U_{n}\right)$}

In what follows we make the following assumptions on $F$ and $g$.

$$
\left\{F_{i}^{\prime}, 1 \geqslant 1\right\} \text { is uniformly bounded in a neighborhood of } \theta(F)
$$

$$
\int_{-\infty}^{\infty}|t| g(t) d t<\infty
$$

Proposition 4.1. Under assumptions in Eqs. (4.1) and (4.2), we have for any $v\left(0<v<\frac{1}{8}\right)$

$$
\sup _{|x-\theta(F)| \leqslant d_{n}}\left|\hat{F}_{n}(x)-\hat{F}_{n}(\theta(F))-\bar{F}_{n}(x)+\bar{F}_{n}(\theta(F))\right|=O\left(n^{-3 / 4+v}\right)+O\left(\alpha_{n}\right)
$$

a.s. as $n \rightarrow \infty$.

Proof. We proceed as in Theorem 3.2 of Lea and Puri. ${ }^{(6)}$ Suppose that $\left\{F_{i}^{\prime}, i \geqslant 1\right\}$ is uniformly bounded in $(\theta(F)-\delta, \theta(F)+\delta)$ for some $\delta>0$. Note that $|x-\theta(F)| \leqslant d_{n} \Rightarrow|x-\theta(F)| \leqslant \frac{1}{2} \delta$ for large $n$. Now,

$$
\begin{aligned}
\left|E\left(\hat{F}_{n}(x)\right)-\bar{F}_{n}(x)\right| & \leqslant \int_{-\infty}^{+\infty}\left|\bar{F}_{n}(x-t)-\bar{F}_{n}(x)\right| g_{n}(t) d t \\
& \leqslant \int_{|t|<\delta / 2}\left|\bar{F}_{n}(x-t)-\bar{F}_{n}(x)\right| g_{n}(t) d t+\int_{|\ell| \geqslant \delta / 2} g_{n}(t) d t
\end{aligned}
$$

But

$$
\begin{aligned}
\int_{|r| \geqslant \delta / 2} g_{n}(t) d t & =\int_{|y| \geqslant \delta x_{n}^{-1 / 2}} g(y) d y \\
& \leqslant \frac{2 \alpha_{n}}{\delta} \int_{|y| \geqslant \delta \alpha_{n}^{-1 / 2}}|y| g(y) d y=O\left(\alpha_{n}\right) \text { as } n \rightarrow \infty
\end{aligned}
$$

and by Taylor's theorem 


$$
\begin{aligned}
\int_{|f|<\delta / 2} & \left|\bar{F}_{n}(x-t)-\bar{F}_{n}(x)\right| g_{n}(t) d t \\
\quad & \int_{|f|<\delta / 2}\left|\bar{F}_{n}^{\prime}\left(x_{t}\right)\right||t| g_{n}(t) d t \\
& \leqslant \text { const. } \alpha_{n} \int_{-\infty}^{+\infty}|y| g(y) d y=O\left(\alpha_{n}\right) \text { as } n \rightarrow \infty
\end{aligned}
$$

where $x_{t}$ is between $x$ and $t$. Combining Eqs. (4.4) and (4.5), we have

$$
\left|E\left(\hat{F}_{n}(x)\right)-\bar{F}_{n}(x)\right|=O\left(\alpha_{n}\right) \quad \text { as } n \rightarrow \infty
$$

Moreover, for $|x| \leqslant d_{n}$

$$
\begin{aligned}
& \left|\hat{F}_{n}(x)-\hat{F}_{n}(\theta(F))-E\left(\hat{F}_{n}(x)\right)+E\left(\hat{F}_{n}(\theta(F))\right)\right| \\
& =\left|\int_{-\infty}^{+\infty}\left(\widetilde{F}_{n}(x-t)-\widetilde{F}_{n}(\theta(F)-t)-\bar{F}_{n}(x-t)+\bar{F}_{n}(\theta(F)-t)\right) g_{n}(t) d t\right| \\
& \leqslant \int_{|t| \geqslant \delta / 2} g_{n}(t) d t \\
& \quad+\int_{|t|<\delta / 2}\left|\widetilde{F}_{n}(x-t)-\widetilde{F}_{n}(\theta(F)-t)-\bar{F}_{n}(x-t)+\bar{F}_{n}(\theta(F)-t)\right| g_{n}(t) d t
\end{aligned}
$$

But

$$
\begin{aligned}
\int_{|f| \geqslant \delta / 2} g_{n}(t) d t & =\int_{|y| \geqslant \delta / 2} g(y) d y \\
& \leqslant \frac{2 \alpha_{n}}{\delta} \int_{|, y| \geqslant \delta / 2 \alpha_{n}}|y| g(y) d y=O\left(\alpha_{n}\right) \quad \text { as } n \rightarrow \infty
\end{aligned}
$$

and the second term of the last inequality in Eq. (4.7) is bounded by

$$
\sup _{|t-\theta(F)| \leqslant \delta / 2} K_{n}(t)=O\left(n^{-3 / 4+v}\right) \text { a.s. } \quad \text { as } n \rightarrow \infty,
$$

by Lemma 2.1 .

From Eqs. (4.7)-(4.9), it follows that

$$
\begin{gathered}
\sup _{|x-\theta(F)| \leqslant d_{n}} \mid \hat{F}_{n}(x)-\hat{F}_{n}(\theta(F))-E\left(\hat{F}_{n}(x)\right)+E\left(\hat{F}_{n}(\theta(F)) \mid\right. \\
=O\left(n^{-3 / 4+v}\right)+O\left(\alpha_{n}\right) \text { a.s. } \quad \text { as } n \rightarrow \infty
\end{gathered}
$$


The proof now follows from Eqs. (4.6), (4.10), and the following inequality

$$
\begin{aligned}
\mid \hat{F}_{n}(x) & -\hat{F}_{n}(\theta(F))-\bar{F}_{n}(x)+\bar{F}_{n}(\theta(F)) \mid \\
\leqslant & \left|\hat{F}_{n}(x)-\hat{F}_{n}(\theta(F))-E\left(\hat{F}_{n}(x)\right)+E\left(\hat{F}_{n}(\theta(F))\right)\right| \\
& +\left|E\left(\hat{F}_{n}(x)\right)-\bar{F}_{n}(x)+E\left(\hat{F}_{n}(\theta(F))\right)\right|-\hat{F}_{n}(\theta(F)) \mid
\end{aligned}
$$

Theorem 4.1. Suppose that $F_{i}^{\prime \prime}(\theta(F)) \forall i \geqslant 1$ exists and is finite. Then, under the conditions of Theorem 3.1 and Eq. (4.2), we have

$$
\hat{F}_{n}\left(U_{n}\right)=\hat{F}_{n}(\theta(F))+m \frac{\bar{F}_{n}^{\prime}(\theta(F))}{n} \sum_{i=1}^{n} X_{n i}^{*}+R_{n}
$$

where $X_{n i}^{*}$ is defined in Eq. (3.5) and

$$
R_{n}=O\left(n^{-3 / 4+v}\right)+O\left(\alpha_{n}\right) \text { a.s. }
$$

as $n \rightarrow \infty$ and $0<v<\frac{1}{8}$.

Proof. First, using Theorem 3.1, we obtain

$$
\begin{gathered}
\left|U_{n}-\theta(F)\right| \leqslant(1+\varepsilon) 2^{1 / 2} m \sigma n^{-1 / 2}(\log \log n)^{1 / 2} \text { a.s. } \\
\text { as } n \rightarrow \infty, \text { for some } \varepsilon>0
\end{gathered}
$$

Next, by Proposition 4.1, we get

$$
\hat{F}_{n}\left(U_{n}\right)-\hat{F}_{n}(\theta(F))=\bar{F}_{n}\left(U_{n}\right)-\bar{F}_{n}(\theta(F))+O\left(n^{-3 / 4+v}\right)+O\left(\alpha_{n}\right)
$$

a.s. as $n \rightarrow \infty$. Now using Young's form of Taylor's theorem, and Eq. (4.12), we obtain,

$$
\bar{F}_{n}\left(U_{n}\right)-\bar{F}_{n}(\theta(F))=\left(U_{n}-\theta(F)\right) \bar{F}_{n}^{\prime}(\theta(F))+O\left(n^{-1} \log \log n\right) .
$$

The proof follows by using Eqs. (4.13), (4.14), and Lemma 2.4 .

Theorem 4.2. Suppose that $F_{i}^{\prime \prime}(\theta(F))$ exists for $\forall i \geqslant 1$ and is finite, the conditions of Theorem 3.1 and condition Eq. (4.2) are satisfied, $\theta(F)>0$, and $\int_{-\infty}^{\infty} y^{2} g(y) d y<\infty$. Then

$$
\hat{F}_{n}\left(U_{n}\right)=n^{-1} \sum_{i=1}^{n} Y_{n i}+R_{n}
$$


where $Y_{n i}=u\left(\theta(F)-X_{i}\right)+m \bar{F}_{n}^{\prime}(\theta(F)) X_{n i}^{*}$ and

$$
R_{n}=O\left(n^{-3 / 4+v}\right)+O\left(n \alpha_{n}^{2} \log \log n\right)^{-1} \text { a.s. }
$$

as $n \rightarrow \infty$ and $0<v<\frac{1}{8}$.

Proof. Applying Theorem 4.1, we obtain

$$
\hat{F}_{n}\left(U_{n}\right)=\hat{F}_{n}(\theta(F))+m \frac{\bar{F}_{n}(\theta(F))}{n} \sum_{i=1}^{n} X_{n i}^{*}+R_{n}
$$

where

$$
R_{n}=O\left(n^{-3 / 4+v}\right)+O\left(\alpha_{n}\right) \text { a.s. as } n \rightarrow \infty
$$

Next using Lemma 2.2, we obtain

$$
\hat{F}_{n}(\theta(F))=\widetilde{F}_{n}(\theta(F))+O\left(n^{-3 / 4+v}\right)+O\left(n \alpha_{n}^{2}(\log \log n)^{-1}\right) \text { a.s. }
$$

The proof follows using Eqs. (4.16) and (4.17).

Theorem 4.3. Under the conditions of Theorem 4.2, if $\alpha_{n}=$ $o\left(n^{-3 / 4+v}(\log \log n)^{1 / 2}\right)$ for $0<v<\frac{1}{8}$ and $F^{\prime}(\theta(F))$ exists and is finite, then

$$
\varlimsup n^{1 / 2} \frac{\hat{F}_{n}\left(U_{n}\right)-F(\theta(F))}{\sqrt{2 \sigma_{*}^{2} \log \log n}}=1 \text { a.s. }
$$

where $\left.\sigma_{*}^{2}=\int_{\mathbb{R}} A^{2}(x) F(d x)+2 \sum_{k=1}^{\infty} \int_{\mathbb{R}^{2}} A(x) A(y) F_{k}^{*} d x, d y\right)$ and $A(x)=$ $u(\theta(F)-x)+m F^{\prime}(\theta(F))\left[h_{1}(x)-\theta(F)\right], \quad$ and $\quad h_{1}(x)=\int h\left(x, x_{2}, \ldots, x_{m}\right)$ $\prod_{i=2}^{m} d F\left(x_{i}\right)$.

Proof. The proof follows from Theorem 4.2 and Lemma 5.6 (in the Appendix).

\section{APPENDIX}

Let $p \geqslant 2$ and $1 \leqslant i_{1}<i_{2}<\cdots<i_{p}$ be arbitrary integers. For any $j(1 \leqslant j \leqslant p-1), P_{j}^{\left(i_{1}, \ldots, i_{p}\right)}$ is the probability measure defined by

$$
\begin{aligned}
& P_{j}^{\left(i_{1}, \ldots, i_{p}\right)}\left(A^{(j)} \times B^{(p-j)}\right) \\
& \quad=P\left[\left(X_{i_{1}}, \ldots, X_{i_{j}}\right) \in A^{(j)}\right] P\left[\left(X_{i_{j+1}}, \ldots, X_{i_{p}}\right) \in B^{(p-j)}\right]
\end{aligned}
$$

and $P_{0}^{\left(i_{1}, \ldots, i_{p}\right)}$ is the probability measure defined by

$$
P_{0}^{\left(i_{1}, \ldots, i_{p}\right)}\left(A^{(p)}\right)=P\left[\left(X_{i_{1}}, \ldots, X_{i_{p}}\right) \in A^{(p)}\right]
$$


for any $A^{(j)} \in \sigma\left(X_{i_{1}}, \ldots, X_{\left.i_{j}\right)} \quad(1 \leqslant j \leqslant p)\right.$ and any $B^{(p-j)} \in \sigma\left(X_{i_{j+1}}, \ldots, X_{i_{p}}\right)$, $(1 \leqslant j \leqslant p-1)$.

Lemma 5.1. For every $p \geqslant 1$ and $\left(i_{1}, \ldots, i_{p}\right)$ such that $1 \leqslant i_{1}<$ $i_{2}<\ldots<i_{p}$ and any $j(1 \leqslant j \leqslant p-1)$, let $h\left(x_{1}, \ldots, x_{p}\right)$ be a Borel function such that

$$
\int_{\mathbb{R}^{n}}\left|h\left(x_{1}, \ldots, x_{p}\right)\right|^{1+\delta} d P_{j}^{\left(i_{1}, \ldots, i_{p}\right)} \leqslant M
$$

for some $\delta>0$, then

$$
\begin{aligned}
& \left|\int_{\mathbb{R}^{n}} h\left(x_{1}, \ldots, x_{p}\right) d P_{0}^{\left(i_{1}, \ldots, i_{p}\right)}-\int_{\mathbb{R}^{n}} h\left(x_{1}, \ldots, x_{p}\right) d P_{j}^{\left(i_{1}, \ldots, i_{p}\right)}\right| \\
& \quad \leqslant 4 M^{1 /(2+\delta)} \beta^{\delta /(1+\delta)}\left(i_{j+1}-i_{j}\right)
\end{aligned}
$$

As a special case, if $h\left(x_{1}, \ldots, x_{p}\right)$ is bounded, say, $\left|h\left(x_{1}, \ldots, x_{p}\right)\right| \leqslant M^{*}$, then we can replace the right side of Eq. (5.1) by $2 M^{*} \beta\left(i_{j+1}-i_{j}\right)$.

Proof. Follows from Lemma 1 of Yoshihara. ${ }^{(19)}$

Lemma 5.2. Let $\left\{Y_{i}, i \geqslant 1\right\}$ be a sequence of absolutely regular random variables with mean 0 . Assume that $\sup _{i \geqslant 1}\left|Y_{i}\right| \leqslant M_{0}$ and $\sup _{i \geqslant 1}\left[\operatorname{Var} Y_{i}\right]^{1 / 2} \leqslant M$. Then, for any $a(0<a<1)$ and $m \leqslant n$ sufficiently large,

$$
P\left[n^{-1}\left|\sum_{i=1}^{n} Y_{i}\right| \geqslant u\right] \leqslant 2 m \exp \left\{-\frac{a k}{5 M_{0}}\right\}+2 n \beta(m)
$$

where $k=[n / m]$.

Proof. Let $\left\{\tilde{Y}_{i}, i \geqslant 1\right\}$ be a sequence of independent random variables such that for any $i, \widetilde{Y}_{i}$ has the same distribution as $Y_{i}$. Then, from Collomb's ${ }^{(2 b)}$ extension of the Bernstein inequality (see Bennett ${ }^{(1)}$ ), we have for any $I \subset\{1, \ldots, n\}$ such that $\operatorname{card} I=m$

$$
P\left[\left|\sum_{i \in I} \tilde{Y}_{i}\right| \geqslant v\right] \leqslant 2 \exp \left\{-\frac{v k^{-a}}{5 M_{0}}\right\}
$$

Let now $S^{(j)}=Y_{j}+Y_{j+m}+\cdots+Y_{j+k_{j} m}(j=1, \ldots, m)$ where for each $j(1 \leqslant j \leqslant m) k_{j}=k_{j, n}$ is the largest integer for which $j+k_{j} m \leqslant n$. Then

$$
P\left[\left|\sum_{i=1}^{n} Y_{i}\right| \geqslant v\right] \leqslant P\left[\sum_{i=1}^{m}\left|S^{(j)}\right| \geqslant v\right] \leqslant \sum_{i=1}^{m} P\left[\left|S^{(j)}\right| \geqslant m^{-1} v\right]
$$


For each $j(1 \leqslant j \leqslant m-1)$, let $A_{j}$ be the Borel subset of $\mathbb{R}^{k_{j}+1}$ defined by

$$
A_{j}=\left\{\left(y_{0}, \ldots, y_{k_{j}}\right):\left|y_{0}+\cdots+y_{k_{j}}\right| \geqslant m^{-1} v\right\}
$$

and put

$$
g_{j}\left(y_{0}, \ldots, y_{k_{j}}\right)= \begin{cases}1 & \text { if }\left(y_{0}, \ldots, y_{k_{j}}\right) \in A_{j} \\ 0 & \text { otherwise }\end{cases}
$$

Then $\left|g_{j}\left(y_{0}, \ldots, y_{k_{j}}\right)\right| \leqslant 1$ and after iterating $k_{j}+1$ times the inequality of Eq. (5.1) for the particular case $M^{*}=1$, we get

$$
\begin{aligned}
P\left[\left|S^{(j)}\right| \geqslant m^{-1} v\right]= & E\left(g_{j}\left(Y_{j}, \ldots, Y_{j+k_{j} m}\right)\right) \\
\leqslant & \int_{\mathbb{R}^{k_{j}+1}} g_{j}\left(y_{0}, \ldots, y_{k_{j}}\right) d F_{j}\left(y_{0}\right) \cdots d F_{j+k_{j} m}\left(y_{k_{j}}\right) \\
& +2\left(k_{j}+1\right) \beta(m) \\
= & P\left[\left|\sum_{i \in I} \widetilde{Y}_{i}\right| \geqslant m^{-1} v\right]+2\left(k_{j}+1\right) \beta(m) \\
\leqslant & 2 \exp \left\{-\frac{m^{-1} v k_{j}^{-a}}{5 M_{0}}\right\} \\
& +2\left(k_{j}+1\right) \beta(m) \text { (from Eq. (5.3)). }
\end{aligned}
$$

Now from Eqs. (5.4) and (5.5), we get

$$
P\left[\left|\sum_{i=1}^{n} Y_{i}\right| \geqslant v\right] \leqslant 2 m \exp \left\{-\frac{u k_{j}^{1-u}}{5 M_{0}}\right\}+2 n \beta(m)
$$

If in Eq. (5.6), we replace $v$ by $u$, we obtain Eq. (5.2).

The following lemma is the Lemma 3 of Chow and Teicher ${ }^{(2 a)}$ [p. 298].

Lemma 5.3. Let $\varphi_{n}^{*}(t)$ be the characteristic function of $S_{n}=\sum_{i=1}^{n} Z_{j}$ where $\left\{Z_{j}, j \geqslant 1\right\}$ are independent (not necessarily stationary) r.v.'s with zero means, and variances $\sigma_{j}^{2}, j \geqslant 1$. If $\Gamma_{n}^{2+\delta}=\sum_{j=1}^{n} \gamma_{j}^{2+\delta}$ and $s_{n}^{2}=\sum_{j=1}^{n} \sigma_{j}^{2}$ where $\gamma_{j}^{2+\delta}=E\left|Z_{j}-E\left(Z_{j}\right)\right|^{2+\delta}$, then for $0 \leqslant \delta \leqslant 1$

$$
\left|\varphi_{n}^{*}\left(\frac{t}{s_{n}}\right)-e^{-t^{2} / 2}\right| \leqslant 3\left|\frac{t \Gamma_{n}}{s_{n}}\right|^{2+\delta} e^{-t^{2} / 2} \quad \text { for } \quad|t|<\frac{s_{n}}{2 \Gamma_{n}}
$$

Lemma 5.4. Let $\left\{Y_{n i}, 1 \leqslant i \leqslant n, n \geqslant 1\right\}$ be a sequence of absolutely 
regular random variables (with means 0 ) satisfying the strong mixing condition. Suppose that $\sum_{m \geqslant 1} \alpha(m)<\infty$ and there exists $\sigma^{2}>0$ such that

$$
\operatorname{Var}\left(\sum_{i=1}^{n} Y_{n i}\right)=n \sigma^{2}(1+o(1))
$$

Then the process $\left\{Y_{n i}, 1 \leqslant i \leqslant n, n \geqslant 1\right\}$ obeys the law of the iterated logarithm, that is $\lim \sup _{n \rightarrow \infty}\left|\sum_{i=1}^{n}\left(Y_{n i} / \chi(n)\right)\right|=1$ a.s., if the following requirements are fulfilled for some $\rho>0$ and for all sufficiently large $n$ :

$$
\sup _{x \in \mathbb{R}}\left|P\left(\sum_{i=1}^{n} Y_{n i}<x \sigma \sqrt{n}\right)-\Phi(x)\right|=O\left(\frac{1}{(\log n)^{1+\rho}}\right)
$$

where

$$
\Phi(x)=\frac{1}{\sqrt{2 \pi}} \int_{-\infty}^{x} e^{-t^{2} / 2} d t
$$

and

$$
P\left[\max _{1 \leqslant j \leqslant n}\left|\sum_{i=1}^{j} Y_{n i}\right| \geqslant b \chi(n)\right]=O\left(\frac{1}{(\log n)^{1+\rho}}\right)
$$

where $b>1$ is an arbitrary number and $\chi(n)=\left(2 \sigma^{2} n \log \log n\right)^{1 / 2}$.

This Lemma was proved by Oodaira and Yoshihara ${ }^{(8)}$ [Th. 1] for the stationary case. The proof is similar for the nonstationary case and is therefore omitted.

Lemma 5.5. For a sequence of random variables satisfying the strong mixing condition, the condition in Eq. (5.10) holds if Eq. (5.9) holds and there exists a function $r=r(n)$ such that $r(n) \leqslant n(n \geqslant 1), r(n) \rightarrow \infty$ as $n \rightarrow \infty$ and

$$
\max \left\{\frac{n}{r} P\left[\sum_{i=1}^{r}\left|Y_{n i}\right| \geqslant \varepsilon \chi(n)\right], \quad \frac{n}{r} \alpha(r)\right\}=O\left(\frac{1}{(\log n)^{1+\rho}}\right)
$$

for any $\varepsilon(0<\varepsilon<(b-1) / b$ where $b>1$ is an arbitrarily fixed number).

Proof. From Oodaira and Yoshihara ${ }^{(8)}$ [Remark 1, Th. 1].

Lemma 5.6. Let $\left\{Y_{n i}, 1 \leqslant i \leqslant n, n \geqslant 1\right\}$ be a sequence of absolutely regular random variables satisfying the strong mixing condition with 
means 0 . Suppose for any $n, m$ such that $n \geqslant m$ and any $J \subset\{1, \ldots, n\}$ such that card $J=m$

$$
E\left(\sum_{j \in J} Y_{n j}\right)^{2}=m \sigma^{2}(1+o(1))
$$

Then the process $\left\{Y_{n i}, 1 \leqslant i \leqslant n, n \geqslant 1\right\}$ obeys the law of the iterated logarithm if the following requirements are fulfilled for some $\delta$ and $\delta^{\prime}$ such that

$$
\begin{gathered}
\sup _{n \geqslant 1} \max _{1 \leqslant i \leqslant n} E\left|Y_{n i}\right|^{2+\delta}=M<\infty \\
\sum_{m=1}^{\infty}\{\alpha(m)\}^{\delta^{\prime}\left(2+\delta^{\prime}\right)}<\infty
\end{gathered}
$$

Proof. Define

$$
f_{N}(x)= \begin{cases}x & \text { if }|x| \leqslant N \\ 0 & \text { otherwise }\end{cases}
$$

We define $\bar{f}_{N}(x)=x-f_{N}(x)$. Let $N=n^{1 / 2\left(1+\delta^{\prime}\right)}(\log n)^{-3}$ and

$$
r(n)=\left[n^{\delta^{\prime} / 2\left(1+\delta^{\prime}\right)}(\log n)^{3}\right]
$$

For convenience and without loss of generality, we denote $Y_{i}$ for $Y_{n i}$. Then for any $b>0$

$$
\begin{aligned}
& \frac{n}{r} P\left[\left|Y_{1}\right|+\cdots+\left|Y_{r}\right| \geqslant b_{\chi}(n)\right] \\
&=\frac{n}{r} P\left[\sum_{i=1}^{r}\left|\bar{f}_{N}\left(Y_{i}\right)\right| \geqslant b \chi(n)\right] \\
& \leqslant \frac{n}{b^{2}\{\chi(n)\}^{2} r} E\left(\sum_{i=1}^{r}\left|\bar{f}_{N}\left(Y_{i}\right)\right|\right)^{2} \\
& \leqslant \frac{n}{b^{2}\{\chi(n)\}^{2} r}\left\{\sum_{1 \leqslant i, j \leqslant r} E\left[\left|\bar{f}_{N}\left(Y_{i}\right)\right| \cdot\left|\bar{f}_{N}\left(Y_{j}\right)\right|\right]\right\} \\
& \leqslant \frac{n}{b^{2}\{\chi(n)\}^{2} r}\left\{2 \sum_{i=0}^{r-1} \sum_{j=1}^{r-i} E\left[\left|\bar{f}_{N}\left(Y_{i}\right)\right| \cdot\left|\bar{f}_{N}\left(Y_{j+i}\right)\right|\right]\right\} \\
& \leqslant \frac{n}{b^{2}\{\chi(n)\}^{2} r}\left\{2 \sum_{i=1}^{r} E\left[\left|\bar{f}_{N}\left(Y_{i}\right)\right|^{2}\right]\right. \\
&\left.+2 \sum_{i=1}^{r-1} \sum_{j=1}^{r-i} E\left[\left|\bar{f}_{N}\left(Y_{i}\right)\right| \cdot\left|\bar{f}_{N}\left(Y_{j+i}\right)\right|\right]\right\}
\end{aligned}
$$




$$
\begin{aligned}
\leqslant & \frac{2 n}{b^{2}\{\chi(n)\}^{2} r}\left\{\frac{1}{N^{\delta}} \sum_{i=1}^{r} E\left[\left|\bar{f}_{N}\left(Y_{i}\right)\right|^{2+\delta}\right]\right. \\
& +12 \sum_{i=1}^{r-1}(\alpha(i))^{\delta^{\prime} /\left(2+\delta^{\prime}\right)} \sum_{j=1}^{r-i} E\left[\left|f_{N}\left(Y_{i}\right)\right|^{2+\delta^{\prime}}\right]^{1 /\left(2+\delta^{\prime}\right)} \\
& \left.\times E\left[\left|\bar{f}_{N}\left(Y_{j+i}\right)\right|^{\left.2+\delta^{\prime}\right)}\right]^{1 /\left(2+\delta^{\prime}\right)}\right\}(\text { from Lemma } 5.7) \\
\leqslant & \frac{2 n}{b^{2}\{\chi(n)\}^{2} r}\left\{\frac{r}{N^{\delta}} M+12 \sum_{i=1}^{r-1}(\alpha(i))^{\delta^{\prime} /\left(2+\delta^{\prime}\right)} \sum_{j=1}^{r-i} \frac{1}{N^{2\left(\delta-\delta^{\prime}\right)}}\right. \\
& \left.\times E\left[\left|\bar{f}_{N}\left(Y_{i}\right)\right|^{2+\delta^{\prime}}\right]^{1 /\left(2+\delta^{\prime}\right)} E\left[\left|\tilde{f}_{N}\left(Y_{j+i}\right)\right|^{2+\delta^{\prime}}\right]^{1 /\left(2+\delta^{\prime}\right)}\right\} \\
\leqslant & \frac{2 n}{b^{2}\{\chi(n)\}^{2} r}\left\{\frac{r M}{N^{\delta}}+\frac{12 r \sum_{i=1}^{r-1}(\alpha(i))^{\delta^{\prime} /\left(2+\delta^{\prime}\right)}}{N^{2\left(\delta-\delta^{\prime}\right)}} M^{2 /\left(2+\delta^{\prime}\right)}\right\} \\
= & O\left(n^{-\gamma}\right)
\end{aligned}
$$

holds for some $\gamma>0$ and

$$
\frac{n}{r} \alpha(r)=\frac{n}{r} O\left(r^{-\left(2+\delta^{\prime}\right) / \delta^{\prime}}\right)=O\left(\frac{1}{(\log n)^{3}}\right)
$$

From Lemmas 5.4 and 5.5 it suffices to show

$$
\sup _{x \in \mathbb{R}}\left|P\left(\sum_{i=1}^{n} Y_{i}<\sqrt{n} \sigma x\right)-\Phi(x)\right|=O\left(\frac{1}{(\log n)^{3}}\right)
$$

Define $p, q$ and $k$ by $p(n)=\left[n^{1 / 2+\infty}\right], q(n)=\left[n^{1 / 2-\infty}\right]$ and $k(n)=[n /(p+q)]$ where $\alpha$ is a small number. Let $N^{\prime}=n^{\delta^{\prime} / 16\left(1+\delta^{\prime}\right)}$ if $0 \leqslant \delta \leqslant 2$ and $N^{\prime}=n^{1 / 16\left(1+\delta^{\prime}\right)}$ if $\delta>2$. Put

$$
\begin{aligned}
& S_{n}^{\prime}=\frac{1}{\sigma \sqrt{n}} \sum_{j=1}^{n}\left(f_{N^{\prime}}\left(Y_{j}\right)-E\left(f_{N^{\prime}}\left(Y_{j}\right)\right)\right) \\
& S^{\prime \prime}=\frac{1}{\sigma \sqrt{n}} \sum_{j=1}^{n}\left(\bar{f}_{N^{\prime}}\left(Y_{j}\right)-E\left(\bar{f}_{N^{\prime}}\left(Y_{j}\right)\right)\right) \\
& Z_{i}=\sum_{j=1}^{p}\left(f_{N^{\prime}}\left(Y_{(i-1)(p+q)+j}\right)\right)-E\left(f_{N^{\prime}}\left(Y_{(i-1)(p+q)+j}\right)\right), \quad i=1, \ldots, k \\
& T_{n}^{\prime}=\frac{1}{\sqrt{n} \sigma} \sum_{i=1}^{k} Z_{i} \\
& T_{n}^{\prime \prime}=S_{n}^{\prime}-T_{n}^{\prime}
\end{aligned}
$$


Then, it can easily be proved that for some $\gamma>0$

$$
E\left|S_{n}^{\prime \prime}\right|^{2} \leqslant \frac{1}{\sigma^{2}}\left(\frac{1}{N^{\prime \delta}} M+\frac{K}{N^{2\left(\delta-\delta^{\prime}\right)\left(2+\delta^{\prime}\right)}} M^{2 /\left(2+\delta^{\prime}\right)}\right)=O\left(n^{-\gamma}\right)
$$

(where $M$ is defined in Eq. (5.13)), $K$ is some constant $>0 . E\left|T_{n}^{\prime \prime}\right|^{2}=O\left(n^{-\gamma}\right)$, $\left|E\left(T_{n}^{\prime 2}\right)-1\right|=O\left(n^{-\gamma}\right)$.

Now, let $\varphi_{n}(t)$ be the characteristic function of $S_{n} / \sigma \sqrt{n}$ where $S_{n}=\sum_{i=1}^{n} Y_{i}$. Then

$$
\begin{aligned}
\left|\varphi_{n}(t)-e^{-t^{2} / 2}\right| \leqslant & \left|\varphi_{n}(t)-E\left(e^{i t S_{n}^{\prime}}\right)\right|+\left|E\left(e^{i t S_{n}^{\prime}}\right)-E\left(e^{i r T_{n}^{\prime}}\right)\right| \\
& +\left|E\left(e^{i t T_{n}^{\prime}}\right)-\prod_{j=1}^{k} E\left(e^{i t Z_{j} / \sqrt{k E\left(Z_{j}\right)^{2}}}\right)\right| \\
& +\left|\left(e^{-t^{2} / 2}\right)-\prod_{j=1}^{k} E\left(e^{i t Z_{j} / \sqrt{k E\left(Z_{j}\right)^{2}}}\right)\right| \\
= & I_{1}+I_{2}+I_{3}+I_{4}, \text { say. }
\end{aligned}
$$

Then, we have

$$
I_{1} \leqslant|t| E\left(\left|S_{n}^{\prime \prime}\right|\right)=O\left(n^{-\gamma / 2}\right), \quad I_{2} \leqslant|t| E\left(\left|T_{n}^{\prime \prime}\right|\right)=O\left(n^{-\gamma / 2}\right)
$$

From Lemma 5.3, we have

$$
\begin{aligned}
& \left|e^{-t^{2} / 2}-\prod_{j=1}^{k} E\left(e^{i t Z_{j} / \sqrt{k E\left(Z_{j}\right)^{2}}}\right)\right| \\
& \leqslant 3 \frac{|t|^{2+\delta}}{k^{\delta / 2}} e^{-t^{2} / 2} \sup _{1 \leqslant j \leqslant k} E\left|Z_{j}\right|^{2+\delta} \min _{1 \leqslant j \leqslant k} \frac{1}{\left(E\left|Z_{j}\right|^{2}\right)^{(2+\delta) / 2}} \\
& \quad \text { for }|t| \leqslant \frac{\left(\sum_{j=1}^{k} E\left(Z_{j}\right)^{2}\right)^{1 / 2}}{2 \sum_{j=1}^{k} E\left|Z_{j}\right|^{2+\delta}}
\end{aligned}
$$

Since

$$
\begin{aligned}
E\left(Z_{j}^{4}\right) & \leqslant K\left(N^{\prime}\right)^{4} p^{2} \sum_{j=1}^{p} j \alpha(j) \leqslant K\left(N^{\prime}\right)^{4} p^{2} \sum_{j=1}^{p} j^{-2 / \delta^{\prime}} \\
& \leqslant K\left(N^{\prime}\right)^{4} p^{2} \max \left(1, p^{-2 / \delta}\right)
\end{aligned}
$$

and $E\left(Z_{j}^{2}\right)=p \sigma^{2}(1+o(1)$ ) (from condition Eq. (5.12)), for all sufficiently large $n$, we have

$$
\begin{aligned}
\sup _{1 \leqslant j \leqslant k} E\left|Z_{j}\right|^{2+\delta} \frac{1}{k^{\rho / 2} \min _{1 \leqslant j \leqslant k}\left(E\left(Z_{j}^{2}\right)\right)^{(2+\rho) / 2}} \\
\quad \leqslant \sup _{1 \leqslant j \leqslant k}\left(E\left(Z_{j}^{4}\right)\right)^{(2+\delta) / 4} \frac{2^{(2+\rho) / 2}}{k^{\rho / 2}\left(\rho \sigma^{2}\right)^{(2+\rho) / 2}}=O\left(n^{-\gamma}\right)
\end{aligned}
$$


holds for all sufficiently large $n$ where $\rho=\min (2, \delta)$ and $\gamma$ is a positive number.

We deduce that

$$
\left|e^{-t^{2} / 2}-\prod_{j=1}^{k} E\left(e^{i t Z_{j} / \sqrt{k E\left(Z_{j}\right)^{2}}}\right)\right| \leqslant K^{\prime} n^{-y}|t|^{2+\delta} e^{-t^{2} / 2}
$$

holds for all sufficiently large $n$ and for all $t$ such that

$$
|t| \leqslant \frac{1}{3} n^{1 / 2-1 /(1+2 \delta)} \sigma \times \frac{1}{\min _{1 \leqslant i \leqslant n} E\left|Y_{i}\right|^{2+\delta}}
$$

Now from Eq. (5.14) and using Lemma 5.7, we obtain

$$
\mid E\left(e^{i t T_{n}^{\prime}}\right)-\prod_{j=1}^{k} E\left(e^{i t Z j / \sqrt{k E\left(Z_{j}^{2}\right)}} \mid \leqslant k \alpha(q)=n^{1 / 2-\alpha} o\left(\left(n^{1 / 2-x}\right)^{-\left(2+\delta^{\prime}\right) / \delta^{\prime}}\right)\right.
$$

From Eqs. (5.17)-(5.23) it follows that

$$
\begin{aligned}
& \left|P\left(\sum_{i=1}^{n} Y_{i}<\sqrt{n} \sigma x\right)-\Phi(x)\right| \\
& \leqslant \int_{-(\log n)^{3}}^{(\log n)^{3}}\left|\frac{\varphi_{n}(t)-e^{-t^{2} / 2}}{t}\right| d t+\frac{K_{1}}{(\log n)^{3}} \\
& \quad \leqslant \int_{-(\log n)^{3}}^{(\log n)^{3}} K_{2} n^{-v}|t|^{1+\delta} d t+\int_{-(\log n)^{3}}^{(\log n)^{3}}\left\{E\left(\left|S_{n}^{\prime \prime}\right|\right)+E\left(\left|T_{n}^{\prime \prime}\right|\right)\right\} d t \\
& \quad+K_{3}\left\{\int_{0 \leqslant|t| \leqslant n^{-1 / 4}} d t+\int_{n^{-1 / 4} \leqslant|t| \leqslant(\log n)^{3}} \frac{k \alpha(q)}{|t|} d t+\frac{K_{4}}{(\log n)^{3}}\right\} \\
& =O\left(\frac{1}{(\log n)^{3}}\right)
\end{aligned}
$$

where $K_{i}, i=1, \ldots, 4$ are constants $>0$, and Lemma 5.6 is proved.

Lemma 5.7. Let $\left\{Y_{n i}, 1 \leqslant i \leqslant n, n \geqslant 1\right\}$ be a sequence of random variables satisfying the strong mixing condition with coefficient $\alpha(m)$. Let $U$ be $\sigma\left(Y_{n i}, 1 \leqslant i \leqslant j \leqslant n\right)$-measurable and $V$ be $\sigma\left(Y_{n i}, j+m \leqslant i \leqslant n\right)$ measurable. If $E|U|^{2}<\infty$ and $E|V|^{2}<\infty$ where $r^{-1}+p^{-1}+s^{-1}=1$ $(1 \leqslant r, s, p<\infty)$ then,

$$
|E(U V)-E(U) E(V)| \leqslant 12(\alpha(m))^{p^{-1}}\left\{E|U|^{r}\right\}^{r^{-1}}\left\{E|V|^{s}\right\}^{s^{-1}}
$$

Proof. This is Proposition 2.8 of Doukhan and Portal. ${ }^{(3)}$ 


\section{REFERENCES}

1. Bennett, G. (1962). Probability inequalities for the sum of independent random variables. J. Amer. Statist. Assoc. 57, 33-45.

2a. Chow, Y. S. and Teicher, H. (1978). Probability Theory. Springer Verlag.

2b. Collomb, G. (1984). Propriétés de convergence presque complète du prédicteur à noyau. Z. Wahrsch. Verw. Gebiete 66, 441-460.

3. Doukhan, P. and Portal, F. (1987). Principe d'invariance faible pour la fonction de répartition empirique dans un cadre multidimensionnel et mélangeant. Prob. and Math. Stat. 8, (2), 117-132.

4. Harel, M. and Puri, M. L. (1991). Théorème central limite de l'estimateur de densité et de l'erreur quadratique intégrée pour des variables aléatoires nonstationnaires. C.R.Academy of Sciences Série 1, 312, 145-148.

5. Harel, M. and Puri, M. L. (1989). Weak convergence of the $U$-statistic and weak invariance of the one sample rank order statistic for Markov processes and ARMA models. J. of Mult. Anal. 31, 258-265.

6. Lea, C. and Puri, M. L. (1988). Asymptotic properties of perturbed empirical distribution functions evaluated at a random point. J. Statistical Planning and Inference 19, 201-215.

7. Nadaraya, E. A. (1964). Some new estimates for distribution function. Theory Probab. Appl. 9, 497-500.

8. Oodaira, H. and Yoshihara, K. (1971). The law of the iterated logarithm for stationary processes satisfying mixing conditions. Ködai Math. Sem. Rep. 23, 311-334.

9. Puri, M. L. and Ralescu, S. (1983). On Berry-Esseen rates, a law of the iterated logarithm and an invariance principle for the proportion of the sample below the sample mean. J. Multivariate Anal. 14, 231-247.

10. Puri, M. L. and Ralescu, S. (1986). Central limit theorem for perturbed empirical distribution functions evaluated at a random point. J. Multivariate Anal. 16, 273-279.

11. Schuster, E. F. (1969). Estimation of probability density function and its derivatives. Ann. Math. Statist. 40, 1187-1195.

12. Scott, D. W., Tapia, R. A. and Thompson, J. R. (1977). Kernel density estimation revisited. J. Nonlinear Analysis Theory Meth. Applic. 1, 339-372.

13. Sun, S. (1993). Asymptotic behavior of the perturbed empirical distribution function evaluated at a random point for absolutely regular sequences J.Multivariate Anal. (to appear).

14. Watson, G. S. and Leadbetter, M. R. (1964). Hazard analysis II. Ser. A, Sankhyā, 26, 101-116.

15. Wertz, W. (1978). Statistical Density Estimation: A Survey. Vandenhoeck and Ruprecht, Göttingen.

16. Winter, B. B. (1973). Strong uniform consistency of integrals of density estimators. Canad. J. Statist. 1, 247-253.

17. Winter, B. B. (1979). Convergence rate of perturbed empirical distribution functions. J. Appl. Probab. 16, 163-173.

18. Yamato, H. (1973). Uniform convergence of an estimator of a distribution function. Bull. Math. Statist. 15, 69-78.

19. Yoshihara, K. (1976). Limiting behavior of $U$-statistics for stationary absolutely regular processes. Z. Wahrsch. Verw. Gebiete 35, 237-252. 